(C) 2003 American Chemical Society, Organometallics, Vilaca om030478m Supporting Info Page 1

$\cup M \cup 504 \% \mathrm{IV}$

\title{
NEW PERYLENE-SUBSTITUTED ORGANOTRIALKYNYLTIN COMPOUNDS FOR THE PHOTOSENSITIZATION OF TIN DIOXIDE
}

\author{
G. Vilaça, K. Barathieu, B. Jousseaume and T. Toupance* \\ Laboratoire de Chimie Organique et Organométallique, UMR 5802 CNRS, Université Bordeaux I, \\ 351 Cours de la Libération, F33405 Talence Cedex, France. \\ H. Allouchi \\ Laboratoire de Chimie Physique PIMIR, EA 2098, Faculté de Pharmacie, \\ 31 Avenue Monge, F37200 Tours, France
}

\section{Supporting Information}

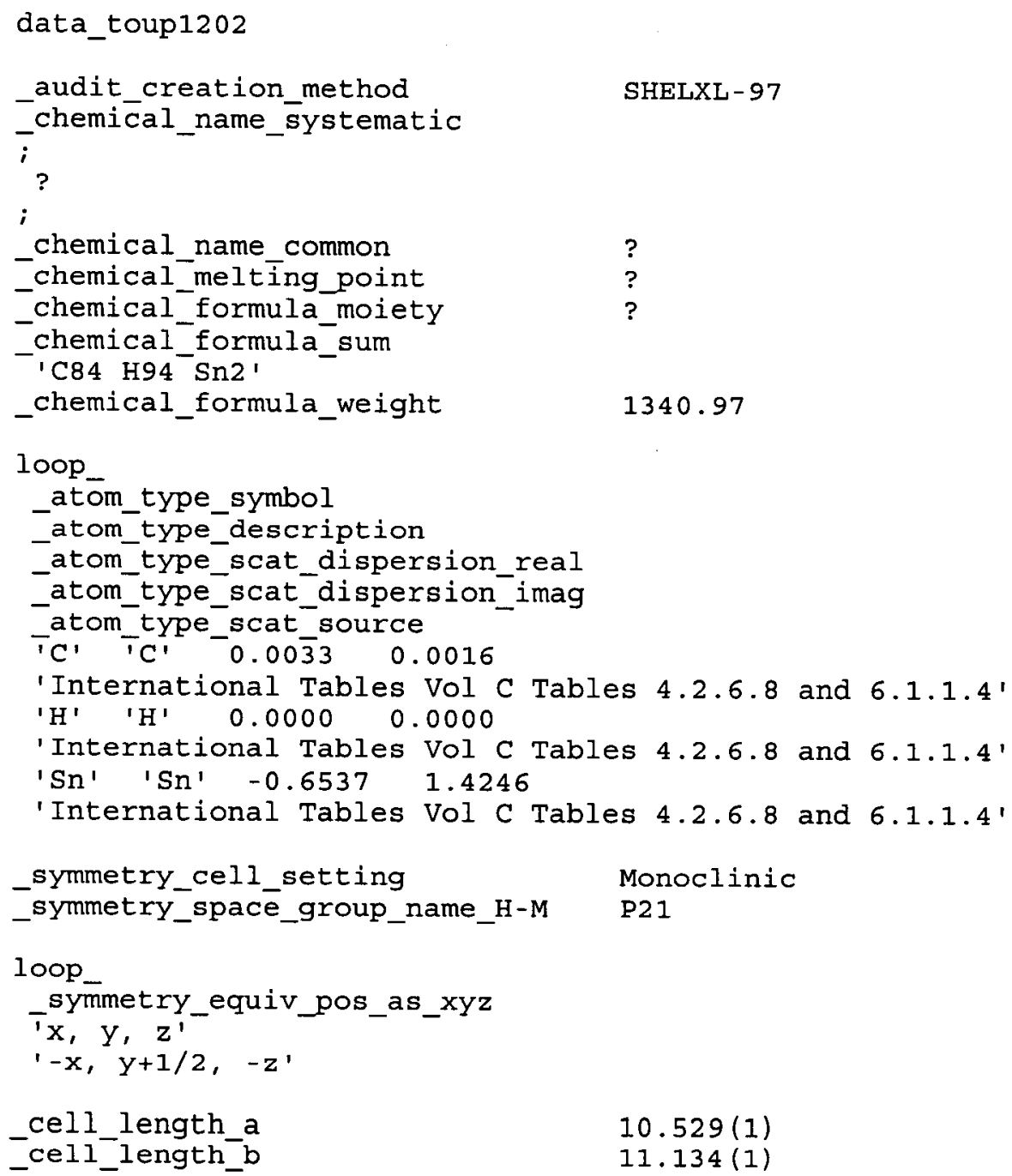




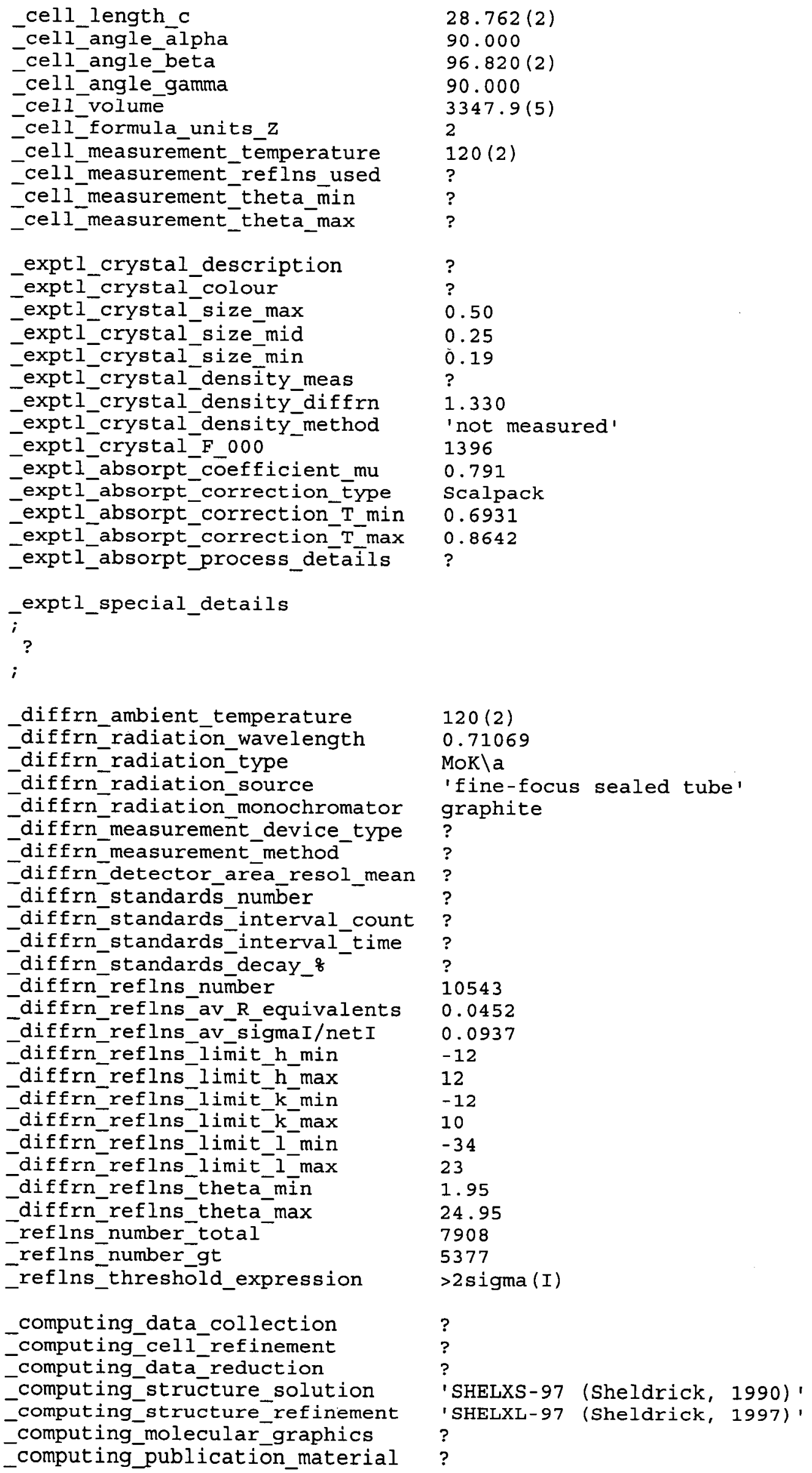




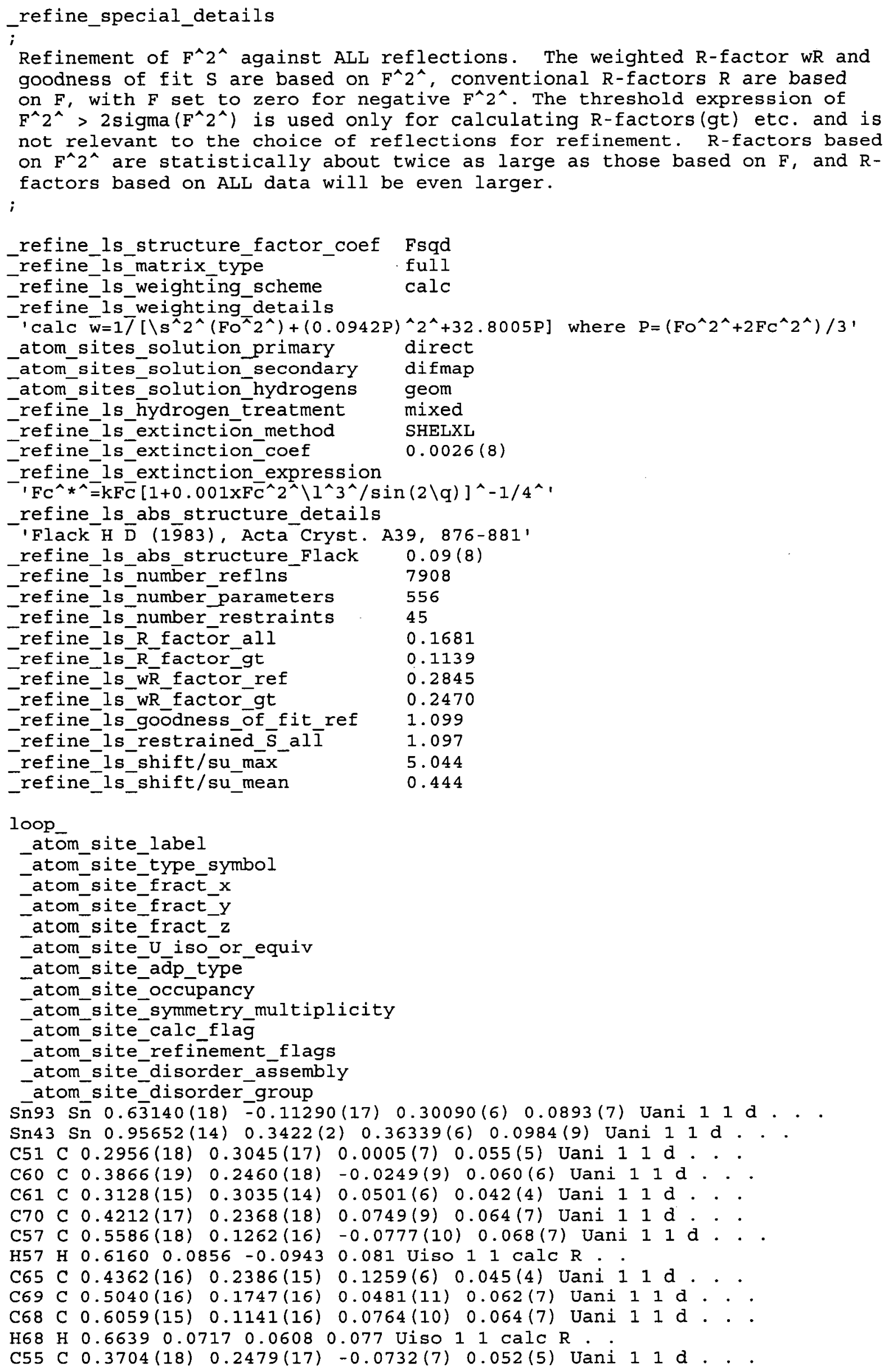


$\begin{array}{lllllllll}C 58 & C & 0.5761(19) & 0.1217(18) & -0.0286(7) & 0.054(5) & \text { Uani } 1 & 1 & d\end{array}$ H58 H $0.64520 .0795-0.01350 .065$ Uiso 1 calc R.

C62 C $0.230(2) \quad 0.362(2) \quad 0.0764(12) \quad 0.078(8) \quad \operatorname{Uani} 11 d$.

H62 H $0.16030 .40390 .0610 \quad 0.094$ Uiso 11 calc R. .

C52 C $0.1927(18) \quad 0.365(2) \quad-0.0223(13) \quad 0.094(9)$ Uani $11 d .$.

H52 H $0.13420 .4030-0.0056 \quad 0.113$ Uiso 11 calc $R$. .

C66 C $0.536(2) \quad 0.180(2) \quad 0.1485(8) \quad 0.073(7) \quad \operatorname{Uani} 11 \mathrm{~d} D$.

C64 C $0.348(2) \quad 0.300(2) \quad 0.1507(11) \quad 0.101(10)$ Uani $11 d . .$.

H64 H 0.35490 .30140 .18320 .121 Uiso 1 1 Calc $R$.

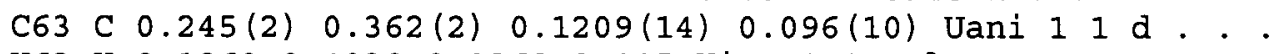

$\mathrm{H} 63 \mathrm{H} \quad 0.1860 \quad 0.40390 .1360 \quad 0.115$ Uiso 1 calc $\mathrm{R}$. .

C59 C 0.4927 (15) $0.1790(15)-0.0022(10) \quad 0.053(6)$ Uani $11 d$. .

C53 C $0.1781(18) \quad 0.367(2) \quad-0.0694$ (13) $0.088(9)$ Uani $11 d$. .

H53 H $0.10910 .4098-0.08410 .105$ Uiso 1 1 Calc R. .

C67 C $0.626(2) \quad 0.113(2) 0.1250(8) \quad 0.068(6)$ Uani $11 \mathrm{~d}$. .

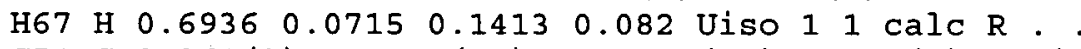

C54 C $0.261(2) \quad 0.3074(16)-0.0993(10) 0.083(7)$ Uani 11 d...

H54 H $0.24540 .3074 \quad-0.13190 .099$ Uiso 11 calc $R$. .

C56 C $0.4612(18) \quad 0.1872(16)-0.1018(9) \quad 0.066(6)$ Uani $11 d$. .

H56 H $0.45130 .1913-0.13440 .079$ Uiso 1 l calc R. .

C20 C $0.8954(15) \quad 0.5537(14) \quad 0.0570(6) \quad 0.039(4)$ Uani 11 d. .

C4 C $0.781(2) \quad 0.4989(19) \quad-0.1175(10) \quad 0.068(7)$ Uani 11 d. .

$\mathrm{H} 4 \mathrm{H} \quad 0.7793 \quad 0.4977 \quad-0.14990 .081$ Uiso 11 calc $\mathrm{R}$.

C11 C $0.8020(15) \quad 0.4959(13) \quad 0.0304(8) \quad 0.040(5)$ Uani $11 d$. .

C13 C $0.713(2) \quad 0.442(2) 0.1021(10) \quad 0.078(7) \quad \operatorname{Uani} 11 \mathrm{~d}$. .

H13 H $0.6487 \quad 0.40530 .11640 .094$ Uiso 1 l calc $\mathrm{R}$.

C1 C $0.7908(16) \quad 0.4948(15) \quad-0.0198(9) \quad 0.053(6)$ Uani 11 d...

C15 C $0.9098(18) \quad 0.5503(17) 0.1068(9) 0.058(6)$ Uani $11 d$. .

C12 C 0.7051 (19) $0.4388(16) \quad 0.0521$ (9) $0.060(6)$ Uani 11 d...

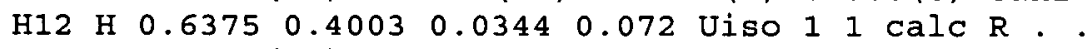

C19 C 0.9967 (15) $0.6119(15) \quad 0.0363(7) \quad 0.045(5)$ Uani $11 \mathrm{~d}$.

C10 C $0.8847(16) \quad 0.5607(15) \quad-0.0432(6) \quad 0.037(4) \quad \operatorname{Uani} 11 \mathrm{~d}$. .

C9 C $0.9857(16) \quad 0.6239(15)-0.0162(5) \quad 0.038(4)$ Uani $11 \mathrm{~d}$. .

C17 C $1.100(2) \quad 0.6728(19) \quad 0.1123(10) \quad 0.067(7)$ Uani $11 \mathrm{~d}$. .

H17 H $1.1668 \quad 0.71330 .12990 .080$ Uiso $1 \quad 1$ calc R. .

C2 C $0.6929(16) \quad 0.4326(14) \quad-0.0503(9) \quad 0.053(6) \quad \operatorname{Uani} 11 \mathrm{~d}$. .

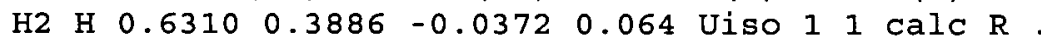

C6 C $0.964(2) \quad 0.630(2) \quad-0.1135$ (9) $0.064(7)$ Uani 11 d . .

H6 H $0.95620 .6326-0.14610 .076$ Uiso 1 1 calc $R$.

C8 C $1.067(2) \quad 0.685(2)-0.0408(8) \quad 0.053(6) \quad \operatorname{Uani} 11 d$.

H8 H $1.13360 .7253-0.02330 .064$ Uiso 1 l calc $\mathrm{R}$. .

C14 C $0.810(2) \quad 0.4940(18) \quad 0.1291(8) \quad 0.063(6)$ Uani 11 d D. .

C7 C $1.0624(16) \quad 0.6930(17)-0.0873(9) \quad 0.053(6)$ Uani 11 d . .

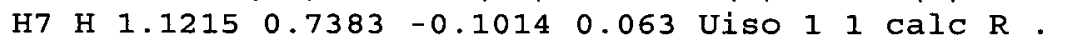

C3 C $0.690(2) \quad 0.4372(19) \quad-0.0987(9) \quad 0.066(6) \quad \operatorname{Uani} 11 d$. .

H3 H $0.6250 \quad 0.3983 \quad-0.11790 .080$ Uiso 1 calc R .

C5 C $0.881(3) 0.566(3)-0.0915(10) 0.044(8)$ Uani 1 id . . .

C18 C $1.090(2) \quad 0.671(2) \quad 0.0634(10) \quad 0.074(7) \quad \operatorname{Uani} 11 d$.

H18 H $1.15130 .71270 .0488 \quad 0.089$ Uiso 1 l calc R

C16 C $1.011(2) \quad 0.6139(17) \quad 0.1332(9) \quad 0.066(7) \quad \operatorname{Uani} 11 d . .$.

H16 H 1.01520 .61500 .16570 .079 Uiso 11 calc $\mathrm{R}$. .

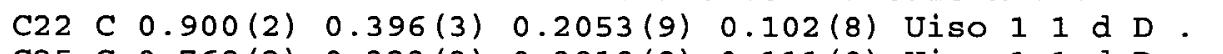

$\begin{array}{llllllllll}\mathrm{C} 25 \mathrm{C} & 0.769(2) & 0.323(3) & 0.3818(9) & 0.111(9) & \text { Uiso } 1 & 1 & \mathrm{~d} & \mathrm{D} & \text {. }\end{array}$

H25 H $0.7350 \quad 0.2524 \quad 0.3640 \quad 0.134$ Uiso 1 calc R . .

C21 C $0.812(2) \quad 0.489(2) 0.1819(9) \quad 0.097(8)$ Uiso 11 d D. .

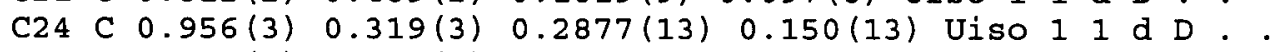

$\mathrm{C} 31 \mathrm{C} 1.060(2) \quad 0.186(2) \quad 0.3886(8) \quad 0.080(7)$ Uiso 11 d D. .

H31 H 1.00680 .11500 .37960 .096 Uiso 11 calc $R$. .

$\mathrm{C} 23 \mathrm{C} 0.881(3) \quad 0.414(3) \quad 0.2585(11) \quad 0.147(13)$ Uiso $11 \mathrm{~d} D$.

C36 C $1.184(3) \quad 0.174(3) \quad 0.3667(10) 0.115(10)$ Uiso 11 d D. .

C76 C $0.5593(19) \quad-0.191(2) 0.3971(7) \quad 0.083(7)$ Uiso $11 \mathrm{~d}$ D. .

H76A H $0.5146-0.26170 .38380 .100$ Uiso 11 calc $R$. .

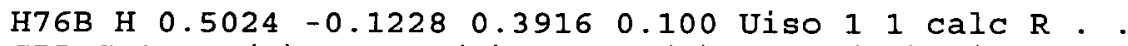

C77 C $0.595(3) \quad-0.209(3) \quad 0.4509(9) \quad 0.126(10)$ Uiso 11 d D. .

H77A H $0.5178 \quad-0.2237 \quad 0.46520 .152$ Uiso 1 calc $R$. .

$\begin{array}{lllllllllll}\mathrm{H} 77 \mathrm{~B} & \mathrm{H} & 0.6488 & -0.2802 & 0.4560 & 0.152 & \mathrm{Uiso} & 1 & 1 \text { calc } \mathrm{R} .\end{array}$ 
C75 C $0.678(2) \quad-0.170(2) \quad 0.3732(8) \quad 0.093(7)$ Uiso 11 d D.. H75 H $0.7297-0.2438 \quad 0.37450 .111$ Uiso 1 calc $R$. . C34 C $1.272(3) \quad 0.075(3) \quad 0.4378(11) \quad 0.129(12)$ Uiso 11 d $\mathrm{D}$. . H34A H $1.31740 .1507 \quad 0.4436 \quad 0.155$ Uiso 11 calc R. .

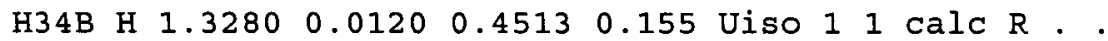
C33 C $1.154(3) \quad 0.078(3) \quad 0.4632(12) \quad 0.128(11)$ Uiso 11 d D.. H33A H $1.17630 .08740 .4967 \quad 0.153$ Uiso 1 l calc $R$. . H33в H $1.1027 \quad 0.0063 \quad 0.4570 \quad 0.153$ Uiso 11 calc R. . C79 C $0.785(2)-0.088(3) \quad 0.4526(8) 0.103(9)$ Uiso 11 d D . . H79A H $0.8390-0.1590 \quad 0.45840 .124$ Uiso 11 calc $R$. .

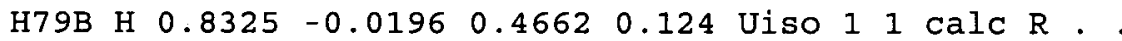
C32 C $1.086(3) \quad 0.189(2) \quad 0.4415(8) \quad 0.103(9)$ Uiso 11 d D. . H32A H 1.00520 .19790 .45420 .123 Uiso 1 l calc $R$. .

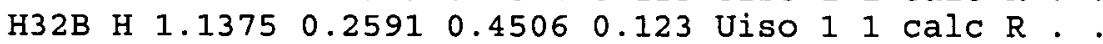
C80 C $0.753(3)-0.070(2) 0.3992(8) 0.111(9)$ Uiso 11 d D.. H80A H $0.7046 \quad 0.00420 .3940 \quad 0.133$ Uiso 11 calc $R$. . H8OB H $0.8326-0.05920 .3857 \quad 0.133$ Uiso 11 calc $R$. . C78 C $0.665(2)-0.104(3) 0.4752(9) \quad 0.105(8)$ Uiso 11 d D..

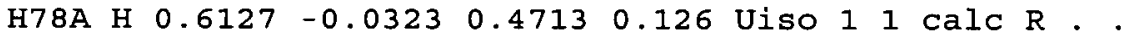

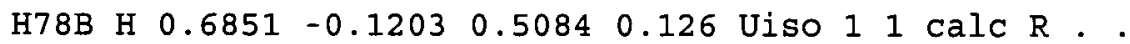
C29 C $0.534(3) \quad 0.401(4) \quad 0.3715(10) 0.139(11)$ Uiso 11 d D. . H29A H $0.47890 .4671 \quad 0.35950 .167$ Uiso 1 l calc $R$. . H29B H $0.5039 \quad 0.3278 \quad 0.3557 \quad 0.167$ Uiso 1 l calc $R$. . $\mathrm{C} 27 \mathrm{C} 0.613(2) \quad 0.281(2) \quad 0.4441(10) \quad 0.100(9)$ Uiso $11 \mathrm{~d} D$. H27A H $0.5750 \quad 0.20810 .43050 .119$ Uiso 1 calc $R$. . H27B H $0.6135 \quad 0.27720 .4778 \quad 0.119$ Uiso 1 l calc $R$. C30 C $0.677(3) \quad 0.427(3) \quad 0.3646(12) \quad 0.126(11)$ Uiso 1 id $\mathrm{D}$. . H30A H 0.70410 .49980 .38140 .151 Uiso 1 l calc $R$. . H30В H $0.68210 .4412 \quad 0.33160 .151$ Uiso 11 calc $R$. . C26 C $0.752(2) \quad 0.294(3) \quad 0.4317(9) \quad 0.105(9)$ Uiso 1 i d D.

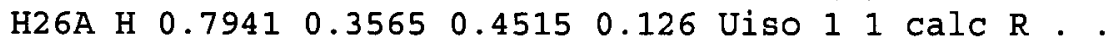
$\begin{array}{llllllllll}\mathrm{H} 26 \mathrm{~B} \mathrm{H} & 0.7967 & 0.2195 & 0.4401 & 0.126 & \text { Uiso } 1 & 1 & \text { calc } \mathrm{R} . & \text {. }\end{array}$ C72 C $0.450(3) 0.090(3) 0.2196(12) 0.145(13)$ Uiso 1 id D.. H72A H $0.4676 \quad 0.0087 \quad 0.2097 \quad 0.174$ Uiso 11 calc R. .

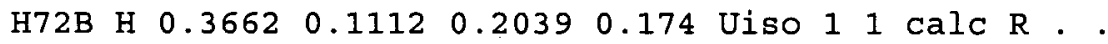
C71 C $0.547(3) \quad 0.172(3) \quad 0.2012(10) \quad 0.135(11)$ Uiso 11 d D. .

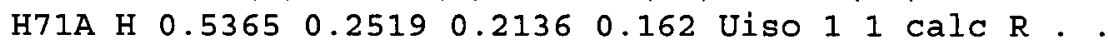

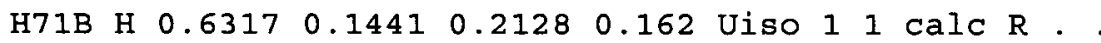
C81 C $0.816(3)-0.126(5) \quad 0.2741(12) \quad 0.166(13)$ Uiso 11 d D. .

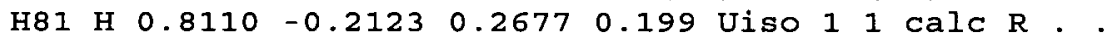
C82 C $0.808(4)-0.088(4) 0.2239(13) 0.183(17)$ Uiso 11 d D.. H82A H $0.7755-0.00630 .22050 .220$ Uiso 11 calc $R$. . H82B H $0.7506-0.1400 \quad 0.2045 \quad 0.220$ Uiso 1 l calc $R$. C83 C $0.945(4)-0.093(5) \quad 0.2075(15) \quad 0.194(18)$ Uiso 11 d D.. H83A H $0.9670 \quad-0.1768 \quad 0.2036 \quad 0.232$ Uiso 11 calc $R$. . $\begin{array}{lllllllll}\text { H83B H } & 0.9415 & -0.0546 & 0.1772 & 0.232 & \text { Uiso } 1 & 1 & \text { calc } R . .\end{array}$ C84 C $1.047(4) \quad-0.036(4) 0.2402(16) \quad 0.196(19)$ Uiso 1 I d D..

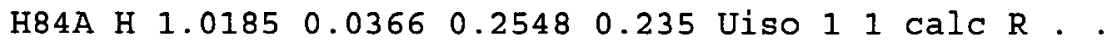
H84B H $1.1243-0.01990 .22600 .235$ Uiso 11 calc $R$. C85 C $1.058(5)-0.143(5) \quad 0.272(2) \quad 0.25(2)$ Uiso 11 d D. . H85A H $1.1399-0.14290 .2918 \quad 0.300$ Uiso 11 calc $R$. . H85B H $1.0496-0.2170 \quad 0.2546 \quad 0.300$ Uiso I I calc $R$. C86 C $0.944(4) \quad-0.130(10) \quad 0.304(2) \quad 0.37(4)$ Uiso $11 \mathrm{~d} D$.

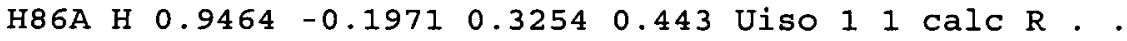

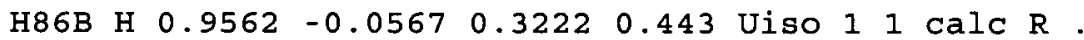
C74 C $0.556(3) \quad 0.062(3) \quad 0.3042(12) 0.129(11)$ Uiso $11 \mathrm{~d} D$. . H74A H 0.53820 .07660 .33610 .154 Uiso 1 l calc $R$. . H74B H $0.62090 .1188 \quad 0.2974 \quad 0.154$ Uiso 1 l calc $\mathrm{R}$. . C87 C $0.507(3) \quad-0.248(3) \quad 0.2612(13) \quad 0.133(11)$ Uiso 11 d D. . $\begin{array}{llllllllll}H 87 & \mathrm{H} & 0.4992 & -0.2293 & 0.2277 & 0.160 & \text { Uiso } 1 & 1 & \text { calc } \mathrm{R} . .\end{array}$ C88 C $0.569(3) \quad-0.372(2) \quad 0.2703(10) \quad 0.103(8)$ Uiso 11 d D. . H88A H $0.5651-0.3980 \quad 0.3023 \quad 0.123$ Uiso 11 calc $R$.

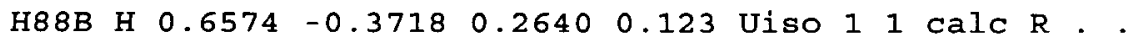
C90 C $0.347(4)-0.468(4) \quad 0.2441(17) \quad 0.176(17)$ Uiso 11 d D. . H90A H $0.3405-0.5054 \quad 0.2742 \quad 0.211$ Uiso 11 calc $R$. . H90B H $0.2981-0.51450 .21970 .211$ Uiso 11 calc $R$. . 
C89 C $0.486(4)-0.455(4) \quad 0.2350(16) 0.20(2)$ Uiso 11 d D. .

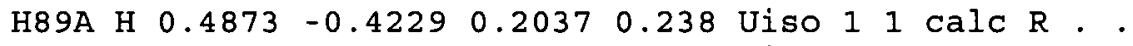
H89B H $0.5243 \quad-0.5339 \quad 0.23590 .238$ Uiso 1 l calc $R$. . C92 C $0.374(3)-0.250(4) 0.2780(13) 0.158(14)$ Uiso 11 d D. . H92A H $0.3356-0.17040 .27600 .189$ Uiso 1 l calc $R$. . H92B H $0.3785-0.27830 .30990 .189$ Uiso 11 calc $R$. C91 C $0.300(4)-0.337(4) 0.2437(17) \quad 0.22(2)$ Uiso 11 d D.. H91A H $0.2117-0.33750 .25010 .269$ Uiso 1 calc $R$. . H91B H $0.3013-0.3058 \quad 0.21230 .269$ Uiso 11 calc $R$. . C38 C $0.991(5) \quad 0.626(4) \quad 0.396(2) \quad 0.32(2)$ Uiso 11 d D. . H38A H $0.9126 \quad 0.61010 .4100 \quad 0.379$ Uiso 1 I calc $R$. .

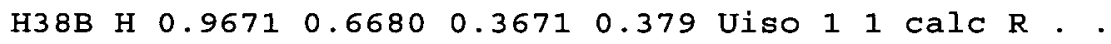
C37 C $1.049(4) \quad 0.508(4) 0.3856(16) 0.158(14)$ Uiso 11 d D. . H37 H $1.0705 \quad 0.48380 .41830 .190$ Uiso 1 l calc $R$. . C28 C $0.535(3) \quad 0.389(3) \quad 0.4249(10) 0.131$ (11) Uiso 11 d D.. H28A H $0.5710 \quad 0.46150 .4400 \quad 0.157$ Uiso 1 l calc $R$. .

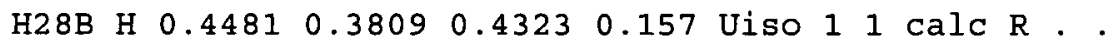
C73 C $0.439(4) \quad 0.085(5) \quad 0.2723(13) \quad 0.189(18)$ Uiso 11 d D.. H73A H $0.4036 \quad 0.1611 \quad 0.28120 .227$ Uiso 1 l calc R . .

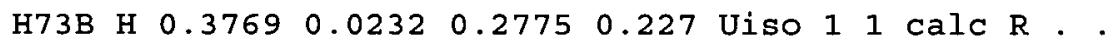
C41 C $1.233(5) \quad 0.637(4) \quad 0.3823(18) 0.22(2)$ Uiso 11 d D. . H4 IA H $1.19090 .68320 .3563 \quad 0.267$ Uiso 1 l calc $R$. . H41B H $1.3236 \quad 0.6374 \quad 0.3786 \quad 0.267$ Uiso 1 calc $R$. . C35 C $1.250(4) \quad 0.056(3) \quad 0.3857(13) 0.183(17)$ Uiso 1 id D.. H35A H $1.1942-0.01270 .37810 .219$ Uiso 1 calc $R$. . H35B H 1.32960 .04330 .37290 .219 Uiso 1 l calc $R$. . C39 C $1.072(5) \quad 0.713(6) \quad 0.430(2) \quad 0.24(3)$ Uiso $11 \mathrm{~d} D$. H39A H $1.0580 \quad 0.69470 .46150 .291$ Uiso 11 calc $R$. . H39B H 1.04140 .79430 .42290 .291 Uiso 11 calc $R$. . C40 C $1.220(5) \quad 0.711(5) \quad 0.4265(18) \quad 0.24(2)$ Uiso 11 d D. . H4OA H $1.2526 \quad 0.79150 .4236 \quad 0.284$ Uiso 1 l calc $R$. . H40B H 1.26490 .67290 .45390 .284 Uiso 1 l calc $R$. . C42 C $1.189(4) \quad 0.508(4) \quad 0.3739(18) \quad 0.19(2)$ Uiso 1 i d D. .

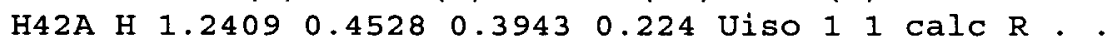
H42B H $1.19230 .48420 .3416 \quad 0.224$ Uiso 1 l calc $\mathrm{R}$. .

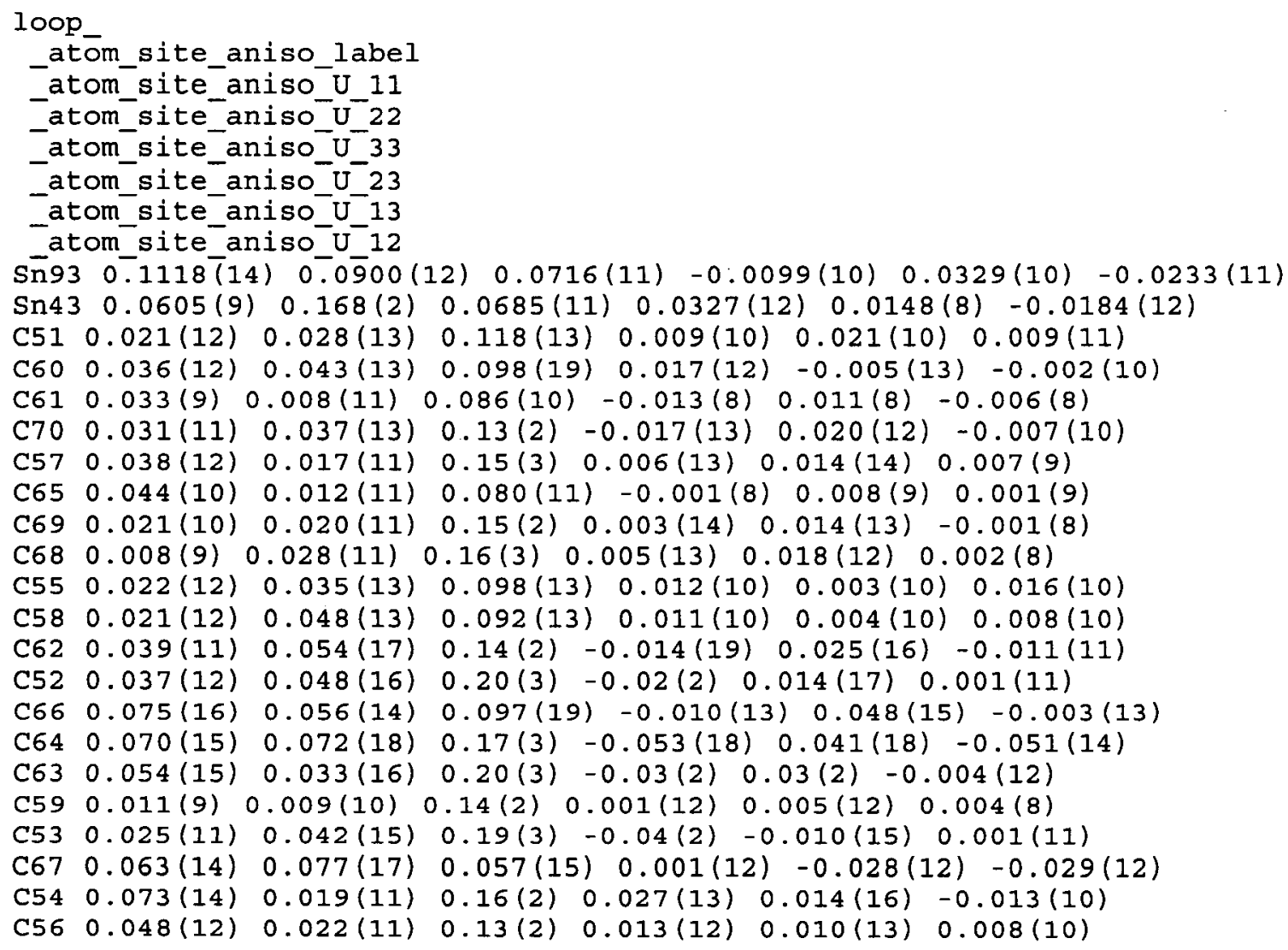




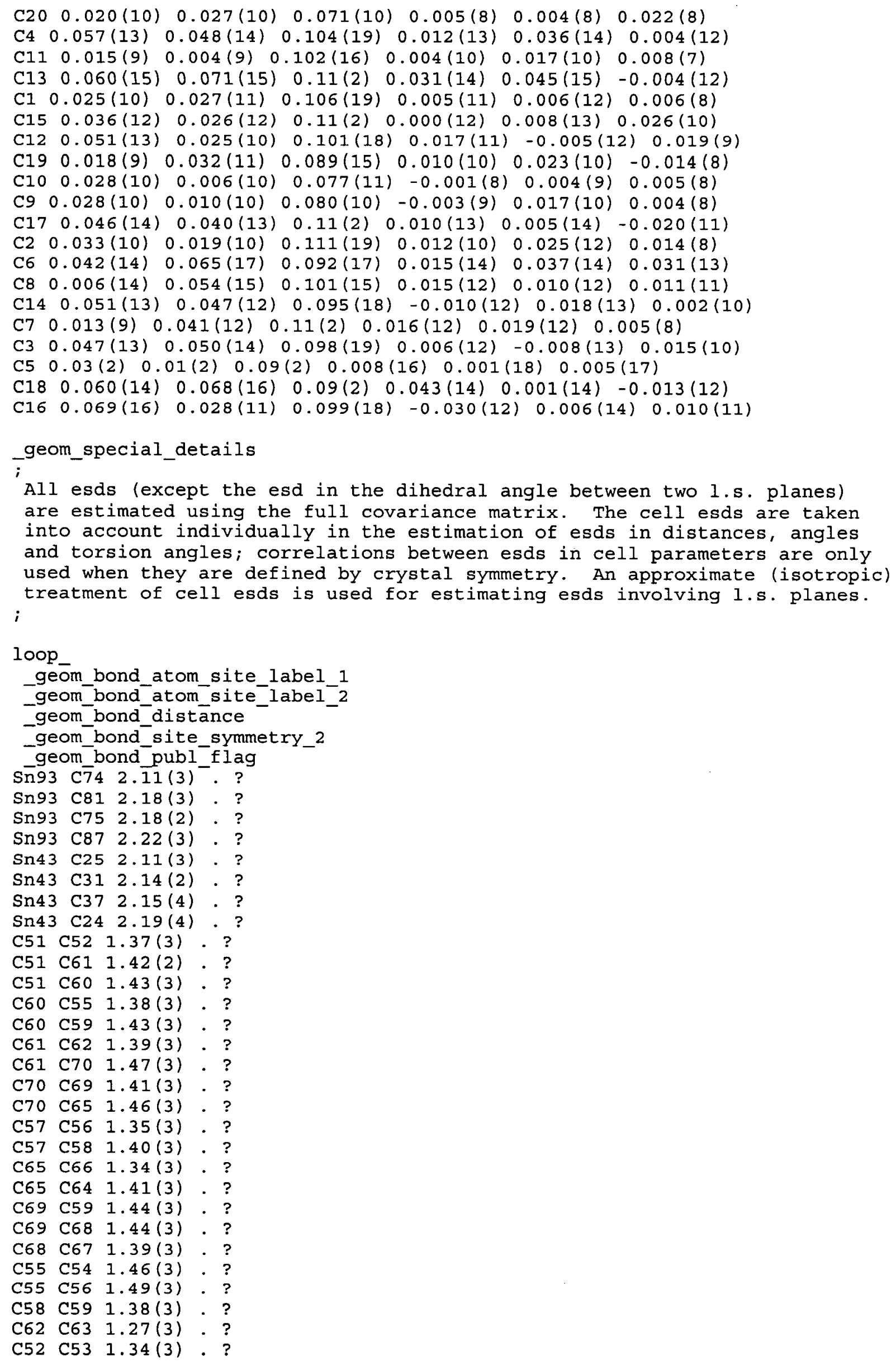




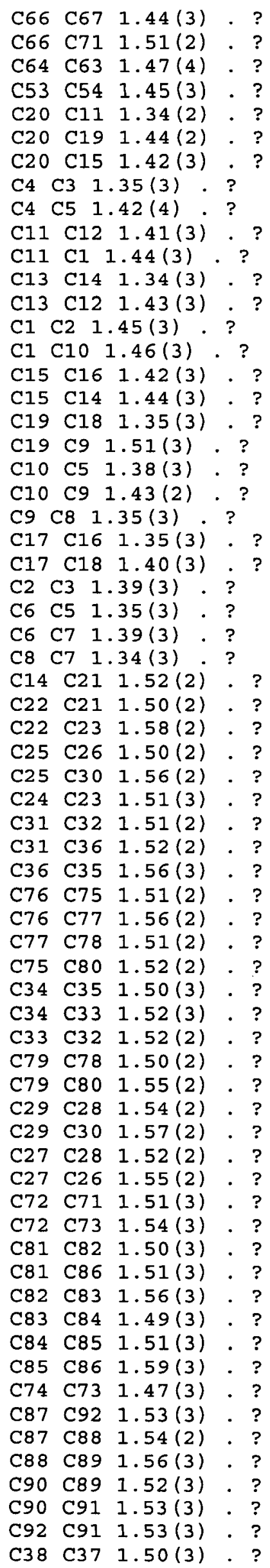




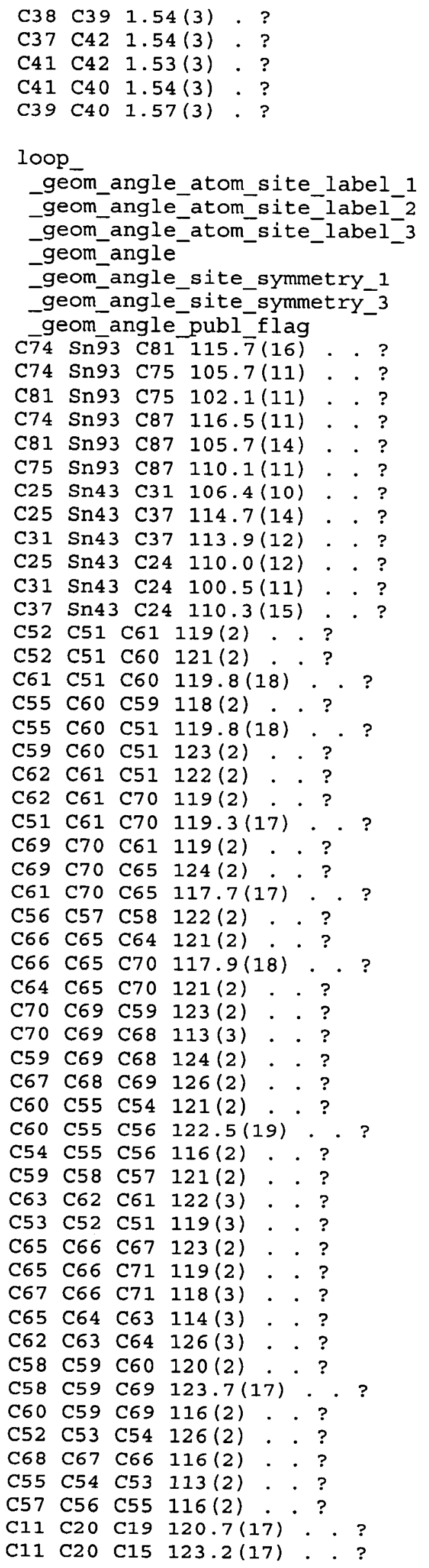




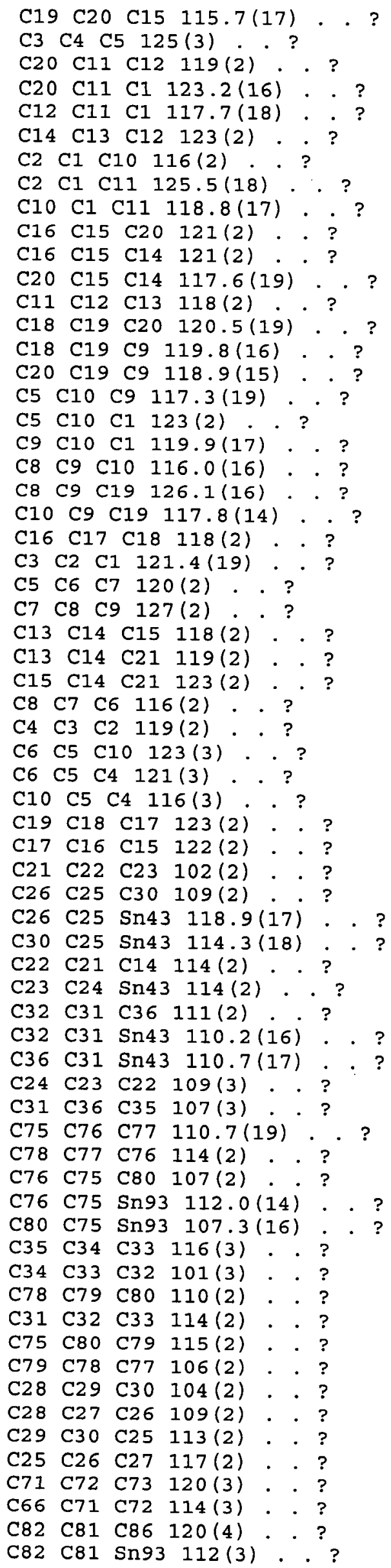




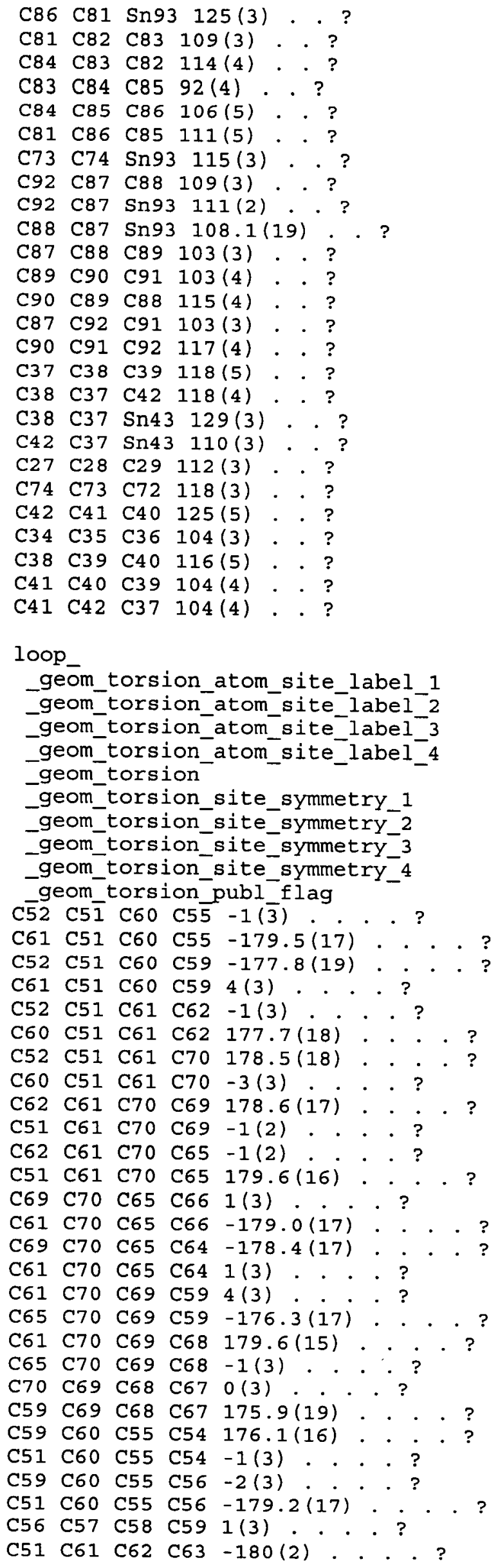




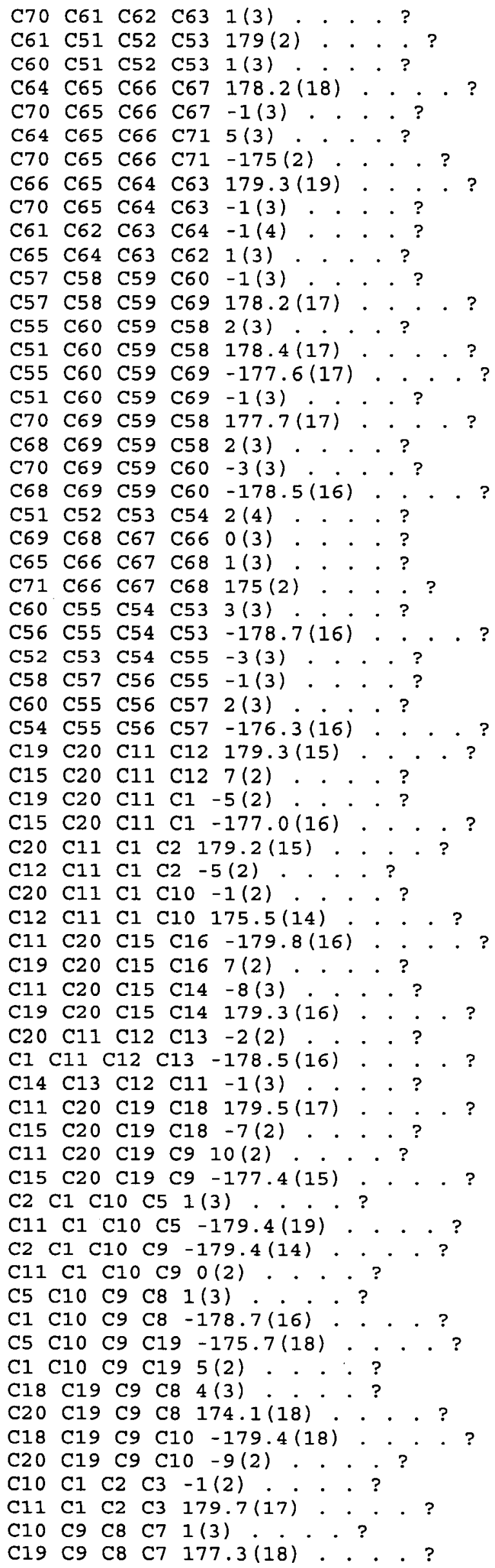




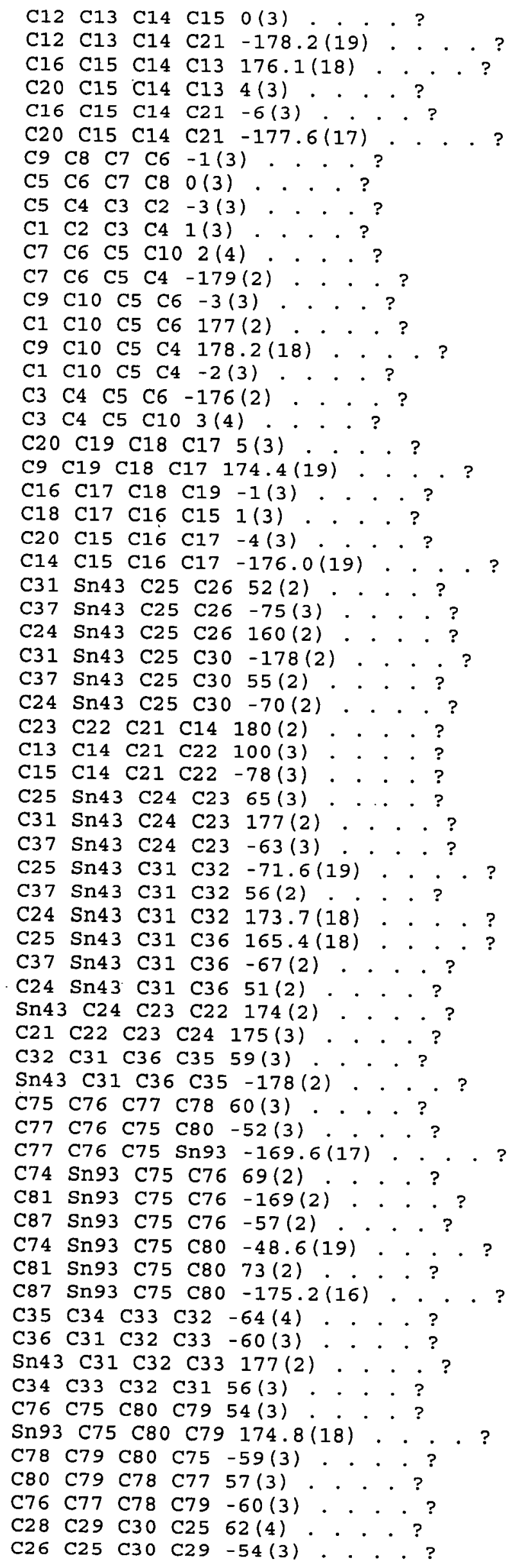




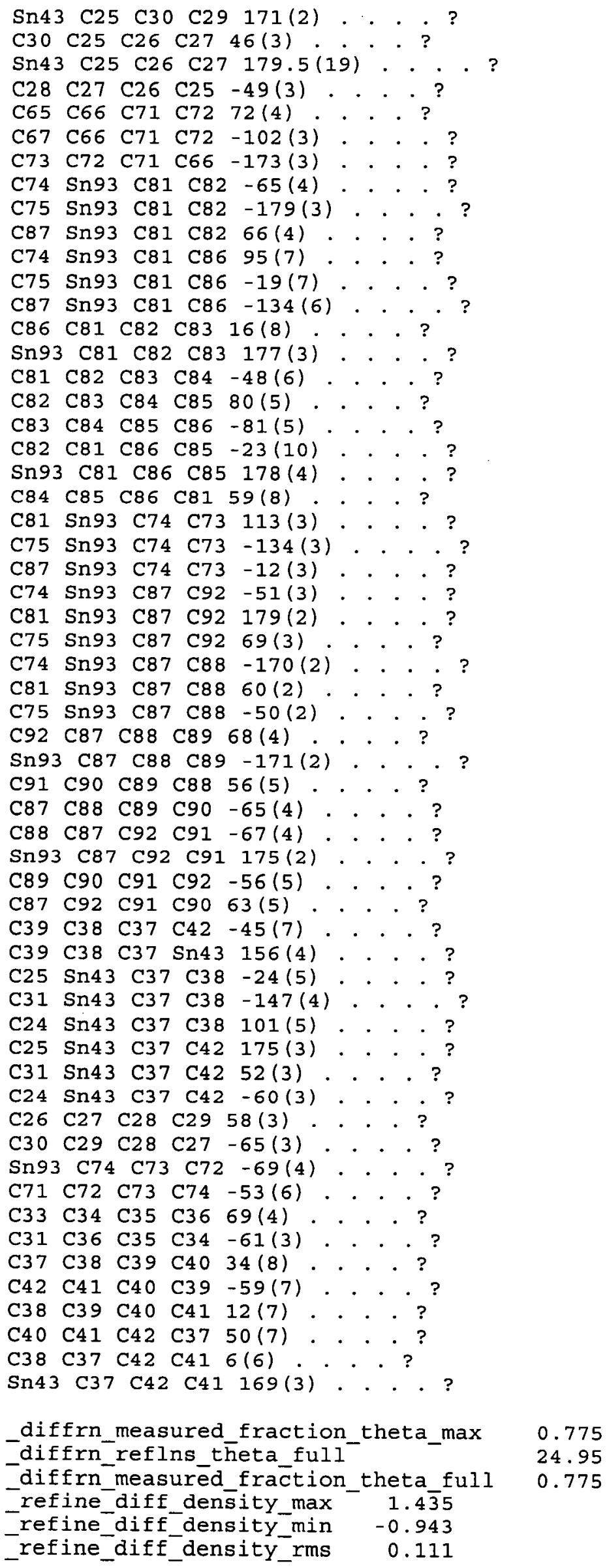

